\title{
BMPR2 Promoter Variants Effect Gene Expression in Pulmonary Arterial Hypertension Patients
}

\author{
Jie Song ${ }^{1,2,3,4}$, Katrin Hinderhofer ${ }^{1}$, Lilian T. Kaufmann ${ }^{1}$, Nicola Benjamin ${ }^{3,4}$, \\ Christine Fischer ${ }^{1}$, Ekkehard Grünig ${ }^{3,4}$ and Christina A. Eichstaedt 1,3,4,*(D) \\ 1 Laboratory for Molecular Genetic Diagnostics, Institute of Human Genetics, Heidelberg University, \\ Im Neuenheimer Feld 366, 69120 Heidelberg, Germany; jie.song@csu.edu.cn (J.S.); \\ katrin.hinderhofer@med.uni-heidelberg.de (K.H.); lilian.kaufmann@med.uni-heidelberg.de (L.T.K.); \\ christine.fischer@med.uni-heidelberg.de (C.F.) \\ 2 Department of Cardiovascular Medicine, The Second Xiangya Hospital of Central South University, \\ Changsha 410011, China \\ 3 Centre for Pulmonary Hypertension, Thoraxklinik gGmbH Heidelberg at Heidelberg University Hospital, \\ Röntgenstrasse 1, 69126 Heidelberg, Germany; nicola.benjamin@med.uni-heidelberg.de (N.B.); \\ ekkehard.gruenig@med.uni-heidelberg.de (E.G.) \\ 4 Translational Lung Research Centre Heidelberg (TLRC), German Centre for Lung Research (DZL), \\ 69120 Heidelberg, Germany \\ * Correspondence: christina.eichstaedt@med.uni-heidelberg.de; Tel.: +49-6221-396-1221; \\ Fax: +49-6221-396-1222
}

Received: 4 September 2020; Accepted: 3 October 2020; Published: 6 October 2020

\begin{abstract}
Pathogenic variants have been identified in $85 \%$ of heritable pulmonary arterial hypertension (PAH) patients. These variants were mainly located in the bone morphogenetic protein receptor 2 (BMPR2) gene. However, the penetrance of BMPR2 variants was reduced leading to a disease manifestation in only $30 \%$ of carriers. In these PAH patients, further modifiers such as additional pathogenic BMPR2 promoter variants could contribute to disease manifestation. Therefore, the aim of this study was to identify BMPR2 promoter variants in PAH patients and to analyze their transcriptional effect on gene expression and disease manifestation. BMPR2 promoter variants were identified in PAH patients and cloned into plasmids. These were transfected into human pulmonary artery smooth muscle cells to determine their respective transcriptional activity. Nine different BMPR2 promoter variants were identified in seven $\mathrm{PAH}$ families and three idiopathic $\mathrm{PAH}$ patients. Seven of the variants (c.-575A > T, c.-586dupT, c.-910C > T, c.-930_-928dupGGC, c.-933_-928dupGGCGGC, c.-930_-928delGGC and c. $-1141 \mathrm{C}>\mathrm{T}$ ) led to a significantly decreased transcriptional activity. This study identified novel $B M P R 2$ promoter variants which may affect BMPR2 gene expression in PAH patients. They could contribute to disease manifestations at least in some families. Further studies are needed to investigate the frequency of $B M P R 2$ promoter variants and their impact on penetrance and disease manifestation.
\end{abstract}

Keywords: BMPR2 promoter; pathogenic variant; heritable pulmonary arterial hypertension

\section{Introduction}

Pulmonary arterial hypertension (PAH) is a rare disease characterized by remodeling of the small pulmonary vessels. This results in an increase of pulmonary artery pressure and resistance, eventually progressing to right heart failure [1]. In many forms of PAH, such as heritable (HPAH) or idiopathic PAH (IPAH), genetic defects have been identified [2]. In most cases of HPAH pathogenic variants (mutations) have been identified in the bone morphogenetic protein receptor 2 (BMPR2) gene leading to a loss of gene function [3]. The BMPR2 gene encodes a cell membrane type II receptor of the transforming growth factor- $\beta$ signaling pathway, which regulates expression of many target genes [4]. 
More than 600 pathogenic variants in the BMPR2 gene have been identified so far, accounting for the disease development in about $85 \%$ of HPAH and $5-35 \%$ of IPAH patients [3]. These patients usually show an earlier onset of disease manifestation, more impaired hemodynamics [2] and reduced survival, compared to $\mathrm{PAH}$ patients without a genetic predisposition [5]. Apart from pathogenic variants in the BMPR2 gene, genetic changes have also been described in recent years in the genes of its coreceptors $A C V R L 1$ and $E N G$, in the gene responsible for pulmonary venous occlusive disease EIF2AK4, and further BMPRII pathway genes [3]. So far, 17 genes have been classified as PAH-causing genes by the international task force for genetics and genomics in PAH [6].

While pathogenic variants in the BMPR2 gene can lead to PAH, an incomplete penetrance of around $30 \%$ has been observed in family members with the same BMPR2 variant [7]. In some families, up to $50 \%$ of $B M P R 2$ variant carriers can develop PAH [8]. It remains unclear why some variant carriers develop PAH while other carriers remain healthy for their entire life. It has been suggested that PAH patients could carry additional pathogenic variants in contrast to their healthy family members $[9,10]$. These so called "second hits" could serve as modifiers for disease penetrance leading to the disease manifestation together with the familial BMPR2 variant [9]. Such second hits were also shown to be present in the noncoding or regulatory regions of the BMPR2 gene [11]. However, in the normal routine genetic diagnostics setting, deep intronic regions and promoters are rarely investigated. Nevertheless, they can also contain pathogenic variants leading to PAH manifestation [12,13]. In previous studies, single substitution variants [14,15], a double substitution variant [13] and deleted or inserted tandem repeats $[14,16]$ downstream of the transcription start site of the BMPR2 gene were identified and led to reduced gene expression in a functional assessment. The contribution of $B M P R 2$ promoter variants to PAH manifestation, however, still remains unclear.

In this study we investigated whether $B M P R 2$ promoter variants in H/IPAH serve as second hits, by firstly identifying $B M P R 2$ promoter variants in $\mathrm{H} / \mathrm{IPAH}$ patients and secondly, functionally characterizing the effect of the variants on $B M P R 2$ gene expression.

\section{Materials and Methods}

\subsection{Study Population}

PAH patients were clinically diagnosed by right heart catheterization following the guidelines of the European Society of Cardiology and the European Respiratory Society [1,17]. Further clinical examinations included medical history, physical examination, 12-lead electrocardiogram, chest radiograph, lung function testing and echocardiography at rest and during exercise. All patients of this study were either idiopathic or heritable $\mathrm{PAH}$ patients. In addition, healthy family members of $\mathrm{H} / \mathrm{IPAH}$ patients performed echocardiography during exercise on a bicycle ergometer in the supine position. Pulmonary arterial systolic pressure (PASP) was measured with an increasing workload until maximum physical exhaustion was reached. A PASP value of $>40 \mathrm{mmHg}$ was used to define a hypertensive exercise response or a normal exercise response (PASP $\leq 40 \mathrm{mmHg}$ ) $[18,19]$. All subjects gave their informed consent for inclusion before they participated in the study. The study was conducted in accordance with the Declaration of Helsinki, and the protocol was approved by the Ethics Committee of the Medical Faculty of Heidelberg University (Project identification codes 065/2001 and S-426/2017).

\subsection{Genetic Analysis}

Genomic DNA was extracted from peripheral blood by an automated procedure (Autopure, Gentra Puregene Technology, Qiagen, Hilden, Germany). Variants in the BMPR2 gene and the promoter region were identified either by direct Sanger sequencing (ABI 3130 genetic analyzer, ThermoFisher Scientific, Waltham, MA, USA) or by a PAH specific gene panel approach based on next-generation sequencing (Miseq, Illumina, San Diego, CA, USA) followed by Sanger sequencing confirmation [20]. Patients with no pathogenic variants in the BMPR2 gene (RefSeq ID: NM_001204) were further evaluated by multiplex ligand-dependent probe amplification (MLPA) to search for large deletions or 
duplications (P093-C1, MRC-Holland, Amsterdam, the Netherlands). The PAH-specific gene panel of this study included 12 PAH genes (ACVRL1, BMPR1B, BMPR2 including the BMPR2 promoter region, CAV1, EIF2AK4, ENG, KCNA5, KCNK3, SMAD1, SMAD4, SMAD9 and TOPBP1) and 17 further candidate genes as described previously [20]. Apart from coding exons, exon-intron boundaries reaching $20 \mathrm{bp}$ into the introns were also analyzed. Each variant with a minor allele frequency in the database Ensembl $<1 \%$ was sought in the Human Gene Mutation Database and variants of BMPR2, ENG, ACVRL1 and SMAD4 also in the ARUP database. Putative transcription factor-binding sites containing promoter variants were assessed using the software MatInspector (version 3.9, Genomatix $\mathrm{GmbH}$, München, Germany).

\subsection{Plasmid Construction}

Nine mutant 1520 base pair (bp) fragments plus the wild-type BMPR2 promoter region (c.-1502 to c.18) were amplified from genomic DNA of PAH patients using a forward primer with a KpnI restriction site at the $5^{\prime}$ end (5'-GAGGGTACCTCCCAAGCCATGCACATTTG-3') and a reverse primer with a HindIII restriction site at the $3^{\prime}$ end (5' - CGCAAGCTTCTGCAGCGAGGAAGTCATC-3') for cloning. The pGL4.10 (Promega GmbH, Walldorf, Germany) vector was cleaved by the KpnI and HindIII restriction endonucleases and ligated with the ten amplified BMPR2 promoter regions. The pGL4.10 vector included the firefly luciferase reporter gene sequence downstream of the inserted promoter region. The correct insertion of the recombinant gene constructs was confirmed by Sanger sequencing. The wild-type sequence contained no variants and corresponded to the human reference sequence (hg19). The pGL4.10 basic vector without any promoter element served as a negative control.

\subsection{Cell Culture and Luciferase Assay}

Commercial human pulmonary artery smooth muscle cells (PASMCs, Life Technologies GmbH, Darmstadt, Germany) were cultured in Medium 231 (Gibco, Thermo Fisher Scientific, Schwerte, Germany), supplemented with smooth muscle growth supplement (Gibco, Thermo Fisher Scientific, Schwerte, Germany) in a $37^{\circ} \mathrm{C}, 5 \%$ carbon dioxide and $95 \%$ humidified cell culture incubator. Cells of passage 3 to 6 were plated onto 24-well plates at 5000 cells per well. After 24 h of incubation pGL4.10-BMPR2-variant constructs or pGL4.10-BMPR2-wild-type plasmids were added to the cells together with a transfection reagent (Lipofectamine 3000, Thermo Fisher Scientific, Schwerte, Germany). After $48 \mathrm{~h}$ of further incubation cells were lysed. Cleared cell lysates were aliquoted into 96-well plates. The activities of the firefly luciferase and the internal standard renilla luciferase were assayed with a Dual-Luciferase Reporter assay kit (Promega GmbH, Walldorf, Germany) using the auto-injector system Lucy2 Microplate Luminometer (Anthos Mikrosysteme GmbH, Krefeld, Germany). Each transfection was carried out five times and measured in triplicate by luciferase assay.

\subsection{Statistical Analysis}

Data were shown as mean \pm standard deviation. Analysis of variance was performed by SPSS (version 21.0.0, IBM, Amonk, NY, USA), considering a $p$-value $<0.05$ as statistically significant. Gene expression was normalized to the wild-type (corresponding to 1.0). Relative gene expression was calculated by: wild-type = value of every mutant plasmid/value of wild-type plasmid .

\section{Results}

\subsection{Clinical Characteristics}

Seven HPAH families with 53 family members and three IPAH patients were included in this study. The clinical characteristics of seven index patients were summarized in Table 1 . The mean age of HPAH index patients was 34 years, $43 \%$ were female. The hemodynamic parameters measured by right heart catheterization revealed a mean pulmonary arterial pressure of $60.3 \pm 9.4 \mathrm{mmHg}$, a pulmonary 
vascular resistance of $20.2 \pm 5.7$ Wood Units and a reduced cardiac index of $2.1 \pm 0.9 \mathrm{~mL} / \mathrm{min} / \mathrm{m}^{2}$. Of 53 sequenced family members, PASP during exercise was measured in 39 individuals.

Table 1. Clinical characteristics of the index patients from the seven HPAH families at initial diagnosis.

\begin{tabular}{cccc}
\hline Characteristic * $^{*}$ & Mean \pm SD & Min & Max \\
\hline Women [\%] & 43 & & \\
Age at diagnosis [years] & $34.0 \pm 14.2$ & 13 & 56 \\
Heart rate [min ${ }^{-1}$ ] & $88.6 \pm 12.2$ & 68 & 99 \\
Oxygen saturation [\%] & $94.0 \pm 5.0$ & 87 & 98 \\
Mean pulmonary artery pressure [mmHg] & $60.3 \pm 9.4$ & 46 & 70 \\
Pulmonary arterial wedge pressure [mmHg] & $5.0 \pm 1.6$ & 3 & 7 \\
Pulmonary vascular resistance [Wood Units] & $20.2 \pm 5.7$ & 13 & 26 \\
Cardiac index [ml/min/m ${ }^{2}$ ] & $2.1 \pm 0.9$ & 1.3 & 3.6 \\
\hline
\end{tabular}

* Not all measurements were obtained from each patient. SD: standard deviation.

\subsection{Genetic Analysis}

To detect promoter variants that may have an impact on gene expression and disease penetrance, a segment of $1520 \mathrm{bp}$ of the $5^{\prime}$ untranslated region (UTR) upstream region of the BMPR2 gene was analyzed. All subjects were included regardless of whether they carried any other pathogenic variant or variant of uncertain significance (VUS) in the coding area of BMPR2 or any other analyzed PAH gene. In total, nine variants were discovered in the promoter region (Figure 1) of which four had not been described before in PAH patients (Table 2). Four heterozygous nucleotide substitutions (c.-301G>A, c.-575A > T, c.-910C > T and c.-1141C > T) and one heterozygous duplication (c.-933_-928dupGGCGGC) were detected in four HPAH families (see Table 2). Additionally, the previously described c.-669G $>$ A variant was identified in three HPAH families. Among these three families, only two index patients carried the c. $669 \mathrm{G}>\mathrm{A}$ variant, while in the third family, this variant was only present in healthy family members.

In two further IPAH patients, a duplication or a deletion of a three base pair repeat out of a twelve repeat GGC region was identified (c.-930_-928dupGGC and c.-930_-928delGGC). This resulted in 11 and 13 GGC repeats in contrast to 12 GGC repeats in the wild-type sequence. The last variant in the BMPR2 promoter region was a homozygous duplication (c.-586dupT) present in a third IPAH patient. The locations of the nine identified variants are depicted in Figure 1.

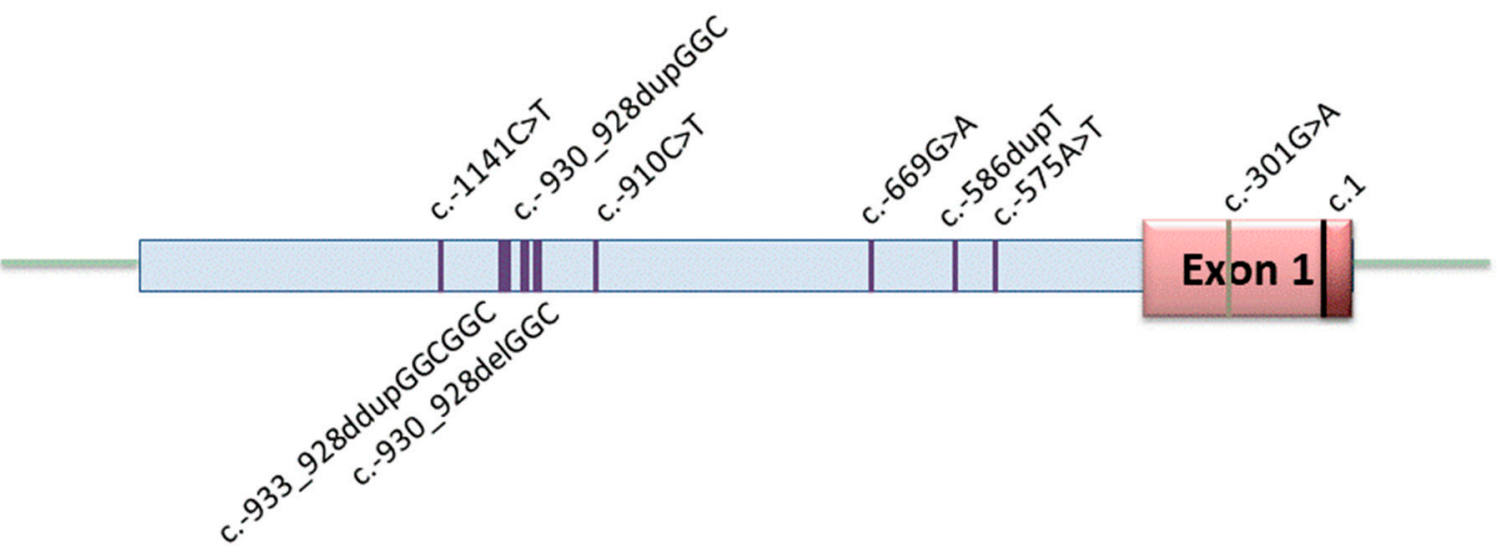

Figure 1. Distribution of variants in the promoter region of BMPR2. Nine variants were identified including five heterozygous substitutions, three heterozygous duplications/deletions at a single site, and one homozygous substitution (c.-586dupT). The $5^{\prime}$ untranslated region starts left of the nucleotide position c.1. 
Table 2. Variants identified in the 5'UTR of the BMPR2 in I/HPAH patients.

\begin{tabular}{|c|c|c|c|c|c|}
\hline Nucleotide Change & Patients & Variant Carriers & GnomAD Frequency & rsID & Described in PAH Patients \\
\hline c. $-301 \mathrm{G}>\mathrm{A}^{1}$ & 2 HPAH families & 2 indices 6 family members ${ }^{2}$ & $0.69 \%$ & rs116154690 & 1 SSc-APAH [15] \\
\hline c. $-575 \mathrm{~A}>\mathrm{T}$ & 1 HPAH family & 1 index & $0.04 \%$ & rs550462760 & This study \\
\hline c.-586dupT ${ }^{3}$ & $1 \mathrm{IPAH}$ patient & 1 index & $0.17 \%$ & rs572725320 & This study \\
\hline c. $-669 \mathrm{G}>\mathrm{A}$ & 3 HPAH families & 2 indices 9 family members & $0.95 \%$ & rs115604088 & {$[11,14]$} \\
\hline c. $-910 \mathrm{C}>\mathrm{T}^{4}$ & 1 HPAH family & 1 index 5 family members & - & - & This study \\
\hline c.-930_-928dupGGC (13 repeats) & $1 \mathrm{IPAH}$ patient & 1 index & - & rs375624016 & 4 CHD-APAH and 10 controls; 1 HPAH $[16,21]$ \\
\hline c.-933_-928dupGGCGGC (14 repeats) ${ }^{4}$ & 1 HPAH family & 1 index 2 family members & - & - & 1 CHD-APAH [16] \\
\hline c.-930_-928delGGC (11 repeats) & $1 \mathrm{IPAH}$ patient & 1 index & - & rs886055459 & 1 CHD-APAH [16] \\
\hline c. $-1141 \mathrm{C}>\mathrm{T}^{1}$ & 1 HPAH family & 1 index 2 family members & - & - & This study \\
\hline
\end{tabular}

${ }^{1}$ c.-301G $>$ A and c.-1141C $>$ T were identified in the same HPAH family but in two different index patients of the same family. ${ }^{2}$ All family members with any BMPR2 promoter variant apart from one c.-301G > A carrier had no manifest PAH. ${ }^{3}$ Homozygous variant, all other variants were heterozygous. ${ }^{4}$ c.-910C $>$ T and c.-933_-928dupGGCGGC were identified in the same HPAH family and index patient. CHD-APAH: congenital heart disease-associated pulmonary arterial hypertension; GnomAD: genome aggregation database; HPAH: heritable pulmonary arterial hypertension; IPAH: idiopathic pulmonary arterial hypertension; rsID: variant identifier; SSc-APAH: systemic sclerosis associated pulmonary arterial hypertension. 


\subsection{Effect of Promoter Variants on Gene Expression}

The effect of the nine different $B M P R 2$ promoter variants on the transcriptional activity of BMPR2 was analyzed using a luciferase reporter gene assay after transient transfection of human PASMCs. The transcriptional activity of the variants was compared to the BMPR 2 wild-type promoter. The results revealed that apart from the two variants c. $-669 \mathrm{G}>\mathrm{A}$ and c.-301G $>\mathrm{A}$, all plasmids with $B M P R 2$ promoter variants led to a statistically significant decrease of transcriptional activity compared to the plasmid carrying BMPR2 wild-type promoter (Figure 2).

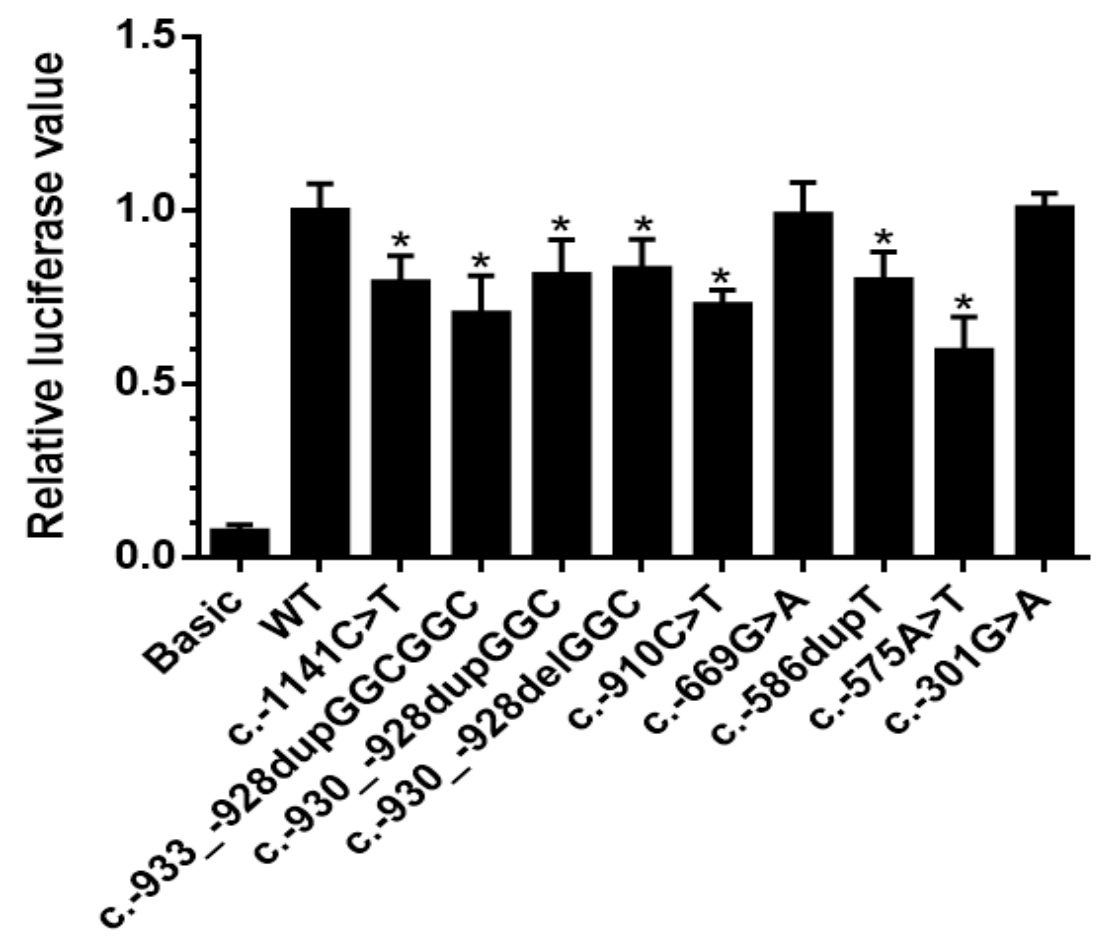

Figure 2. Transcriptional activity analysis of $B M P R 2$ promoter variants. The transcriptional activity of nine $B M P R 2$ promoter variants was compared with the wild-type BMPR2 promoter by dual-luciferase assay. Seven of the nine variants showed significantly reduced gene expression in comparison to the wild-type. Basic: pGL4.10 plasmid (without promoter, negative control), WT: wild-type, c.-930_-928delGGC: 11GGC repeats, c.-930_-928dupGGC: 13GGC repeats, c.-933_-928dupGGCGGC: $14 G G C$ repeats. Data are presented as mean \pm standard error of the mean, normalized to the wild-type; $p$-value refers to the comparison between each mutant to wild-type; ${ }^{*} p<0.05$.

\subsection{Association of Promoter Variants with an Abnormal Pulmonary Artery Pressure during Exercise or PAH Manifestation}

One family (Family 1) was identified with two promoter variants in the index patient (Figure 3). The index patient (II: 2) was a 42-year-old woman, who was diagnosed with PAH 6 years prior to study inclusion. At diagnosis she presented with exertional dyspnea and limited exercise capacity. The echocardiogram revealed an enlarged right ventricle and right atrium. Right heart catheterization showed a mean pulmonary arterial pressure of $67 \mathrm{mmHg}$, pulmonary arterial wedge pressure of $5 \mathrm{mmHg}$, and pulmonary vascular resistance of 26 Wood Units. The patient carried the two variants c.-910C $>$ T and c.-933_-928dupGGCGGC in the BMPR2 promoter region. Each variant was inherited from one parent, thus being biallelic and compound heterozygous in the index patient (Figure 3). Both variants were shown to lead to a reduced gene expression in the functional analysis (Figure 2). Apart from the index patient, her brother (II: 5) also carried both promoter variants but did not develop PAH. The c.-910C $>$ T variant was also found in her mother (I: 2 ) and in four other family members (II: 4, II: 5, III: 4, III: 5) without manifest PAH. In four of the five family members with the c.-910C > T 
variant an elevated PASP during exercise was measured by echocardiography, while only one variant carrier showed a normal pressure response (Figure 3, Family 1 in Table 3). This observation fits to the hypothesis of cosegregation of the variant with an elevated PASP assuming an autosomal dominant model of inheritance with reduced penetrance.

In contrast, the second BMPR2 promoter variant (c.-933_-928dupGGCGGC) showed no cosegregation with abnormal PASP (Figure 3). Thus, while the first variant may be associated with a hypertensive exercise response the second variant does not seem to contribute to disease manifestation.

(a)

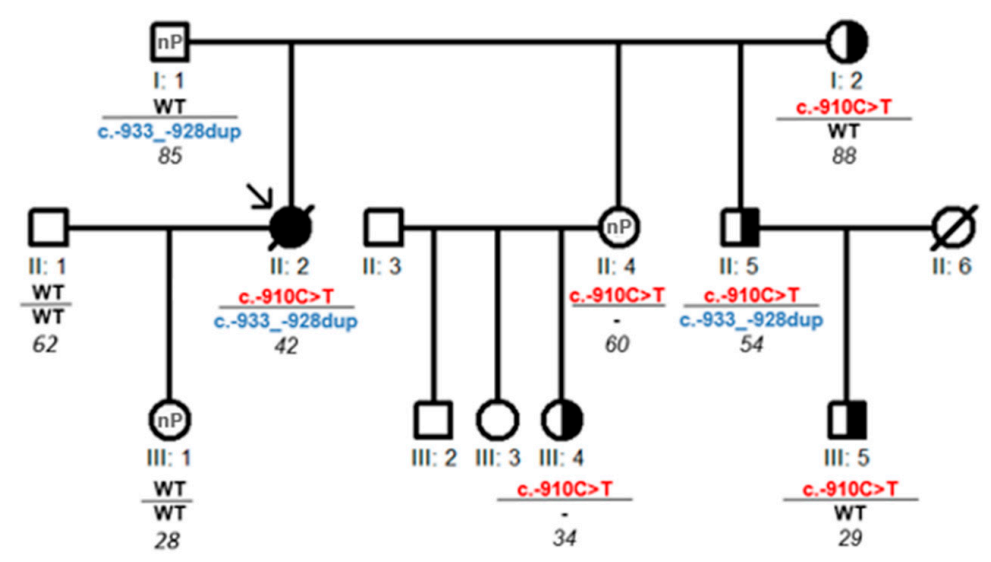

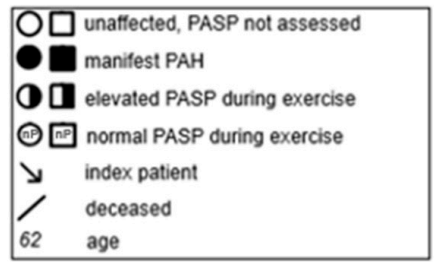

(b)

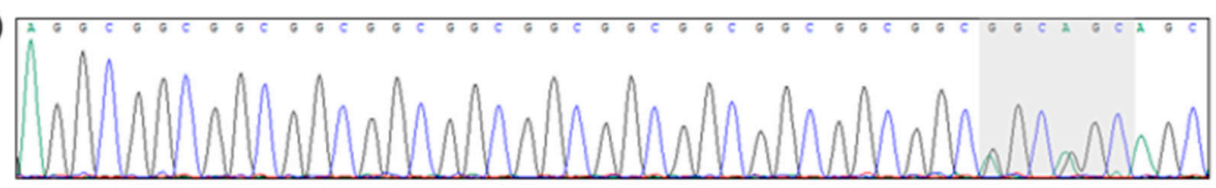

(c)

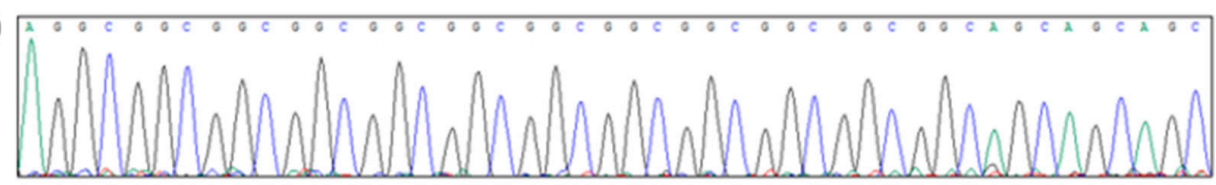

(d)

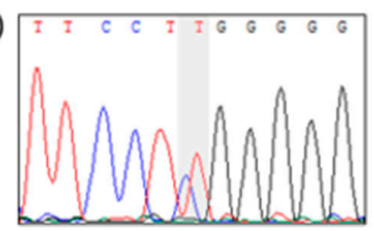

(e)

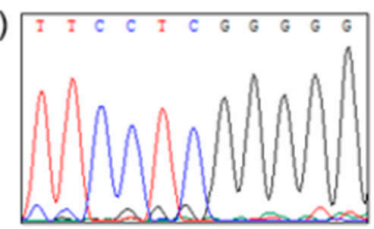

Figure 3. Pedigree of Family 1 with c.-910C $>T$ and c.-933_-928dupGGCGGC in the $5^{\prime} \mathrm{UTR}$ of $B M P R 2$ gene: (a) the pedigree with BMPR2 5'UTR variants c.-910C $>$ T and c.-933_-928dupGGCGGC. Only the c.-910C $>\mathrm{T}$ variant is present in all family members with an elevated pulmonary arterial systolic pressure (PASP) during exercise. The horizontal line separates the two loci in BMPR2 5'UTR c.-910C $>$ T and c.-933_-928dupGGCGGC variants; c.-910C $>$ T: BMPR2 c.-910C $>$ T; c.-933_-928dup: BMPR2 c.-933_-928dupGGCGGC; WT: wild-type; -: not sequenced. Italic numbers represent the age of individuals; (b) sequencing analysis of the c.-933_-928dupGGCGGC shows the heterozygous $\mathrm{G}>\mathrm{A}$ changes on the right side within the grey shaded area indicating a duplication of a $3 \mathrm{bp}$ repeat; (c) c.-933_-928 wild-type sequence; (d) sequencing analysis of the heterozygous c.-910C $>\mathrm{T}$ variant within the grey shaded area; (e) c.-910 wild-type sequence. 
In the other HPAH families no cosegregation of the respective promoter variant with an abnormal PASP was apparent (Table 3). In one family (Family 3, Table 3) the promoter variant (c.-575A $>$ T) was not present in the second HPAH patient of the family. In another family (Family 2, Table 3) a single, different promoter variant was identified in the two HPAH patients of the family. However, one of the two variants (c.-301G>A) revealed no effect on gene expression in the functional analysis. Promoter variants, which reduced gene expression were identified in six of the ten I/HPAH index patients (Table 3). Out of these six patients only three patients had a known pathogenic variant in the coding region of a PAH gene. Thus, in these patients the promoter variant could be considered a possible second hit adding to a known pathogenic variant.

Table 3. BMPR2 promoter and further gene variants in $\mathrm{I} / \mathrm{HPAH}$ patients.

\begin{tabular}{|c|c|c|c|c|c|}
\hline $\begin{array}{l}\text { Family }^{-/-} \\
\text {Index }\end{array}$ & $\begin{array}{l}\text { Other Pathogenic } \\
\text { Variants/VUS }\end{array}$ & $\begin{array}{l}\text { BMPR2 } \\
\text { Promoter } \\
\text { Variants }\end{array}$ & $\begin{array}{c}\text { Gene } \\
\text { Expression } \\
\text { Compared to } \\
\text { Wild-Type }\end{array}$ & $\begin{array}{c}\text { Variant in FM } \\
\text { with } \uparrow \text { Exercise } \\
\text { PASP/all FM } \\
\text { with } \uparrow \text { Exercise } \\
\text { PASP }\end{array}$ & $\begin{array}{c}\text { Variant in FM } \\
\text { with Normal } \\
\text { Exercise } \\
\text { PASP/All FM } \\
\text { with Normal } \\
\text { Exercise PASP }\end{array}$ \\
\hline \multirow{2}{*}{ Family 1} & \multirow{2}{*}{ none } & c. $-910 \mathrm{C}>\mathrm{T}$ & $0.73 x$ & $4 / 4$ & $1 / 2$ \\
\hline & & $\begin{array}{c}\text { c.-933_-928 } \\
\text { dupGGCGGC }\end{array}$ & $0.70 x$ & $1 / 4$ & $1 / 2$ \\
\hline \multirow{2}{*}{ Family 2} & EIF2AK4 c.641delA & c. $-1141 \mathrm{C}>\mathrm{T}$ & $0.79 x$ & \multirow{2}{*}{$\begin{array}{c}\text { in index patient } \\
\text { in } 2 \text { nd PAH } \\
\text { patient }\end{array}$} & NA \\
\hline & p.(Lys214Argfs*21) & c. $-301 G>A$ & $1.01 \mathrm{x}$ & & NA \\
\hline Family 3 & none & c. $-575 \mathrm{~A}>\mathrm{T}$ & $0.60 x$ & NA & $\begin{array}{c}\text { not in } 2 \text { nd PAH } \\
\text { patient }\end{array}$ \\
\hline Family 4 & none & c. $-301 G>A$ & $1.01 \mathrm{x}$ & $1 / 2$ & $1 / 1$ \\
\hline Family $5^{1}$ & $\begin{array}{l}\text { BMPR2 c. } 244 \mathrm{C}>\mathrm{T} \\
\text { p. }\left(\mathrm{G} \ln 82^{*}\right)\end{array}$ & c. $-669 \mathrm{G}>\mathrm{A}$ & $0.99 x$ & $3 / 6$ & $0 / 2$ \\
\hline Family 6 & $B M P R 2$ exon 2-3 deletion & c. $-669 \mathrm{G}>\mathrm{A}$ & $0.99 x$ & $4 / 6$ & $0 / 2$ \\
\hline Family 7 & $\begin{array}{c}\text { ENG c. } 1633 \mathrm{G}>\mathrm{A} \\
\text { p.(Gly545Ser) }\end{array}$ & c. $-669 \mathrm{G}>\mathrm{A}$ & $0.99 x$ & $1 / 1$ & $2 / 5$ \\
\hline IPAH 1 & none & c.-586dupT & $0.80 x$ & NA & NA \\
\hline IPAH 2 & $\begin{array}{c}\text { BMPR2 c. } 1453 \mathrm{G}>\mathrm{A} \\
\text { p.(Asp } 485 \mathrm{Asn})\end{array}$ & c.-930_-928delGGC & $0.83 x$ & NA & NA \\
\hline IPAH 3 & $\begin{array}{c}\text { BMPR2 c. } 2695 \mathrm{C}>\mathrm{T} \\
\text { p. }\left(\operatorname{Arg} 899^{*}\right)\end{array}$ & c.-930_-928dupGGC & $0.82 x$ & NA & NA \\
\hline
\end{tabular}

1 in Family 5, BMPR2 c.-669G $>$ A was identified in healthy family members but not in the index patient. In other families, variants listed were identified in the index patients. Exercise PASP was measured in 39 of 53 sequenced family members. $\uparrow$ : hypertensive; FM: family member; NA: no PASP measured in family members during exercise; PASP: pulmonary arterial systolic pressure; VUS: variants of uncertain significance. Reference sequence IDs: BMPR2: NM_001204,EIF2AK4: NM_001013703,ENG: NM_001114753.

Two of the three IPAH patients, four HPAH patients and one HPAH family member were sequenced with a PAH-specific panel for $12 \mathrm{PAH}$ genes and 17 candidate genes [20]. This identified a novel homozygous pathogenic EIF2AK4 variant c.641delA p.(Lys214Argfs*21) in one family with two affected sisters clinically characterized as HPAH, albeit pulmonary veno-occlusive disease could not be excluded retrospectively. Further variants in the study cohort were reported previously (BMPR2 c.224C $>$ T p.Gln82*; BMPR2 c.1453G > A p.Asp485Asn; BMPR2 c.2695C > T p.Arg899*; BMPR2 exon 2-3 deletion; ENG c.1633G>A p.Gly545Ser) [2,11,20].

\section{Discussion}

This study identified four novel and five further variants in the BMPR2 promoter of I/HPAH patients. The transcriptional impact of these variants revealed a reduced transcriptional activity for seven out of nine variants $(78 \%)$.

\subsection{Impact of Promoter Variants on Transcription Factor Binding Sites}

The variant c.-910C $>\mathrm{T}$ was identified for the first time in this study. This patient harbored not only the c.-910C > T but additionally the c.-933_-928dupGGCGGC variant in the BMPR2 promoter region. 
These two variants were both shown to decrease transcriptional activity of BMPR2. The c.-910C $>\mathrm{T}$ variant was predicted to create a new binding site for the transcription factor Gli-similar 3 (GLIS3). Glis3 is a Krüppel-like transcription factor as a member of Gli and Zinc finger families which shows a tissue specific expression mainly in the endocrinal system such as the thyroid gland and in low levels also in the lung [22]. So far, no study reported the relation of Glis3 and the BMPR2 gene or any PAH pathway related gene. However, the new PAH gene Krüppel-like transcription factor 2 (KLF2) could indicate a possible relation to this Zinc finger family member and PAH pathogenesis [23,24]. The promoter variant c. $-910 \mathrm{C}>\mathrm{T}$ did not cosegregate with PAH in the family but with a hypertensive PASP during exercise. In contrast, the second variant of the index patient in Family 1 (c.-933_-928dupGGCGGC) showed no cosegregation with an abnormal PASP response in family members and was less likely to have contributed to PAH manifestation.

The variant c.-1141C $>\mathrm{T}$ of BMPR2 promoter was also newly identified in a HPAH patient and a significant reduction in the BMPR2 promoter transcriptional activity expression was shown. In addition, in silico a loss of a pleomorphic adenoma gene 1 (PLAG1) transcription factor binding site was predicted. It was reported that $P L A G 1$ could act as an activator for a gene promoter by upregulating the insulin-like growth factor gene IGF1 [25]. However, no study has so far elucidated the relation between the transcription factor PLAG1 and the BMPR2 gene. If it served as an activator of the BMPR2 promoter, the loss of the PLAG1 binding site predicted in this study would be consistent with the measured reduced transcriptional activity. However, the variant showed no cosegregation with the disease or an abnormal PASP response in the family, possibly only attributing a weak functional impact on the BMPR2 gene and PAH manifestation. Reduced transcriptional activity without cosegregation in the family was also identified for the heterozygous variant c. $-575 \mathrm{~A}>\mathrm{T}$.

Moreover, this study identified three different types of GGC repeats in the c.-963_-928 upstream sequence of BMPR2. All of these variants led to a reduced transcriptional activity. The wild-type sequence at position c.-963_-928 upstream of transcription start site is made up of 12 tandem GGC repeats. Limsuwan and colleagues first discovered an abnormal number of GGC repeats within these sequences in congenital heart disease-associated PAH children [16]. The transcriptional assessment of the altered number of GGC tandem repeats in our functional analysis (11, 13 and 14 GGC repeats) in Family 1 and two IPAH patients were in line with another study showing a decrease in gene expression with a promoter containing 13 or 10 GGC repeats [21] in comparison to the 12-GGC repeat. A further study identified a GC>AT change within this repeat sequence cosegregating with the disease in an HPAH family [13]. The authors could establish that the variant led to a preferred cryptic translational start site, followed by a premature stop codon and nonsense mediated decay. This variant could not be identified within the GGC-repeat region in our study.

The "GGCG" sequence is a possible early growth response-1 (EGR1) transcription factor binding site. EGR1 binds to the promoter region of many target genes, thereby participating in various pathways such as those relevant for cardiovascular homeostasis [26]. This factor can serve as an activator or repressor and it can be activated by hypoxia in vitro [26]. Gaddipati et al. demonstrated an increase of EGR1 can lead to a decreased expression of BMPR2 [27]. However, in another study, knockdown of the EGR1 gene by short interfering RNA treatment resulted in a reduction of BMPR2 promoter transcription activity [21]. Thus, the complex molecular mechanisms between the EGR1 and $B M P R 2$ promoter remain to be further investigated.

\subsection{Contradictions to Previous Studies}

The two variants c.-301G > A and c.-669G > A neither indicated a cosegregation with the disease nor showed any influence on gene transcription. This suggests that both variants may be benign. This is supported by the presence of both alleles in non-PAH controls in the gnomAD database (c.-301G > A: $0.69 \%$ in 217 out of 31,336 individuals; c.-669G>A: 0.95\% in 297 out of 31,142 individuals). The functional study results contradicted a previous study, which had shown a reduced transcriptional activity of the c.-669G $>$ A variant [14]. The variant was present in only two index patients of three 
HPAH families in our study. In one family it co-occurred with a pathogenic BMPR2 exon 2-3 deletion and in the second family with a VUS in the ENG gene. Therefore, both the pedigree and functional results suggest a benign nature of the variant, also contradicting our own previous assumptions, which suggested the c.-669G $>$ A variant contributed to disease manifestation [11].

For c.-301G $>$ A, the segregation data and functional analysis showed opposing results to Pousada and colleagues, who reported a decreased gene expression in plasmids harboring the c.-301G $>A$ variant [15]. A predicted loss of an Msh Homeobox 2 (MSX2) transcription factor binding site may result in a reduced $B M P R 2$ expression. So far, no study reported any relation between the MSH2 and $B M P R 2$ expression. A previous study demonstrated in Msx2-/- mice an upregulation of BMPR2 in smooth muscle cells in peripheral arteries [28]. Hence, the contradictory results above require further clarification to determine the effects of the promoter variants on BMPR2 expression levels.

In contrast to the former studies, this study used a longer 5'UTR of the BMPR2 gene as promoter sequence $(1520 \mathrm{bp})$ in order to incorporate all detected variants and be able to compare them with each other in the same experimental set-up. In previous studies shorter $5^{\prime}$ UTR fragments of $300 \mathrm{bp}$ containing the region c.-770_c.-471 [14] and 539 bp including the base pairs c.-539_c.-1 [15] were used. A 5'UTR length-dependent up- or down-regulation of BMPR2 has previously been described due to the inclusion of additional transcriptional modulators [27] and could have led to the different results. The origin of the cell culture cells used in functional analyses could also have influenced the results. In this study, PASMCs were used, which are at the heart of vascular remodeling in PAH, while in one of the two other studies the kidney cell line COS-1 was analyzed [15]. Expression data from different organs furthermore suggest the BMPR2 gene expression in the lungs to be around three times higher than in the kidneys [29]. Thus, the different length of the promoter region encompassing different stretches and transcription factor binding sites of the BMPR2 promotor, the different cell types and organ-specific gene expression may explain the different expression levels of the same variants between published works and this study. In future studies, expression levels should be compared in constructs with similar length of the inserted sequences and the same cell lines to have clearly comparable results to resolve the discrepancies.

\subsection{Limitations and Future Directions}

In this study, we only assessed one defined length of the promoter region in one specific cell line. Analyzing the effect of the promotor variants on BMPR2-gene expression using sequence fragments of different lengths in various cell lines side by side could help to elucidate the interaction of the transcription regulation domains within the promoter. Moreover, two promoter variants were identified in the same patient (Figure 3) but their effect was only analyzed separately. It would be interesting to evaluate their joint effect in the same plasmid and experimental setting to find out whether they are additive, lead to an even greater effect or cancel each other out.

In addition, the DNA-methylation of the promoter region should be measured in future studies to obtain a full picture of transcriptional activity. Equally, the evaluation of the subcellular localization of the respective BMPRII proteins could provide further functional evidence and valuable information regarding genetic variants in the BMPR2 promoter region in future studies.

\section{Conclusions}

New variants in the BMPR2 promoter region were discovered and investigated in this study. A down-regulated gene expression facilitated by variants in the BMPR2 promoter suggested a possible impact on disease manifestation. However, the majority of variants showed no cosegregation with the disease or an elevated PASP during exercise in families, despite a statistically significant transcriptional impact. Thus, the promoter variants cannot fully explain the causality between the clinical manifestation and genotypes. There is still a lack of frequency data for promoter variants in a large PAH dataset. Predicted transcriptional factor binding sites remain to be validated and the complex mechanisms of altering these sites and the influence on gene transcription require further detailed 
investigations to determine the consequences on BMPR2 expression levels. Thus, further studies are needed to investigate the frequency of $B M P R 2$ promoter variants and their impact on penetrance and disease manifestation.

Author Contributions: Conceptualization, J.S., K.H., E.G. and C.A.E.; methodology, J.S., L.T.K., N.B., C.F., E.G., C.A.E.; software, J.S., N.B. and C.F.; validation, J.S. and C.A.E.; formal analysis, J.S., N.B. and C.F.; investigation, J.S., L.T.K. and C.A.E.; resources, K.H., L.T.K. and E.G.; data curation, J.S. and C.A.E.; writing-original draft preparation, J.S. and C.A.E.; writing-review and editing, K.H., L.T.K., N.B., C.F. and E.G.; visualization, J.S. and C.A.E.; supervision, K.H., L.T.K., E.G. and C.A.E.; project administration, C.A.E.; funding acquisition, K.H. and E.G. All authors have read and agreed to the published version of the manuscript.

Funding: This research received no external funding.

Acknowledgments: The authors would like to thank Kirsten Linsmeier and Susanne Theiss from the Institute of Human Genetics at Heidelberg University for their kind technical support.

Conflicts of Interest: The authors declare no conflict of interest.

\section{References}

1. Galiè, N.; Humbert, M.; Vachiéry, J.-L.; Gibbs, S.; Lang, I.M.; Kaminski, K.A.; Simonneau, G.; Peacock, A.; Noordegraaf, A.V.; Beghetti, M.; et al. 2015 ESC/ERS Guidelines for the diagnosis and treatment of pulmonary hypertension. Eur. Hear. J. 2015, 37, 67-119. [CrossRef] [PubMed]

2. Pfarr, N.; Szamalek-Hoegel, J.; Fischer, C.; Hinderhofer, K.; Nagel, C.; Ehlken, N.; Tiede, H.; Olschewski, H.; Reichenberger, F.; Ghofrani, H.A.; et al. Hemodynamic and clinical onset in patients with hereditary pulmonary arterial hypertension and BMPR2 mutations. Respir. Res. 2011, 12, 99. [CrossRef] [PubMed]

3. Machado, R.D.; Southgate, L.; Eichstaedt, C.A.; Aldred, M.A.; Austin, E.D.; Best, D.H.; Chung, W.K.; Benjamin, N.; Elliott, C.G.; Eyries, M.; et al. Pulmonary Arterial Hypertension: A Current Perspective on Established and Emerging Molecular Genetic Defects. Hum. Mutat. 2015, 36, 1113-1127. [CrossRef] [PubMed]

4. Olschewski, A.; Berghausen, E.M.; Eichstaedt, C.A.; Fleischmann, B.K.; Grünig, E.; Grünig, G.; Hansmann, G.; Harbaum, L.; Hennigs, J.K.; Jonigk, D.; et al. Pathobiology, pathology and genetics of pulmonary hypertension: Update from the Cologne Consensus Conference 2018. Int. J. Cardiol. 2018, 272, 4-10. [CrossRef]

5. Evans, J.D.W.; Girerd, B.; Montani, D.; Wang, X.-J.; Galié, N.; Austin, E.D.; Elliott, G.; Asano, K.; Grunig, E.; Yan, Y.; et al. BMPR2 mutations and survival in pulmonary arterial hypertension: An individual participant data meta-analysis. Lancet Respir. Med. 2016, 4, 129-137. [CrossRef]

6. Morrell, N.W.; Aldred, M.A.; Chung, W.K.; Elliott, C.G.; Nichols, W.C.; Soubrier, F.; Trembath, R.C.; Loyd, J.E. Genetics and genomics of pulmonary arterial hypertension. Eur. Respir. J. 2019, 53, 1801899. [CrossRef]

7. Larkin, E.K.; Newman, J.H.; Austin, E.D.; Hemnes, A.R.; Wheeler, L.; Robbins, I.M.; West, J.; Phillips, J.A.; Hamid, R.; Loyd, J.E. Longitudinal Analysis Casts Doubt on the Presence of Genetic Anticipation in Heritable Pulmonary Arterial Hypertension. Am. J. Respir. Crit. Care Med. 2012, 186, 892-896. [CrossRef]

8. Frydman, N.A.; Steffann, J.; Girerd, B.; Frydman, R.; Munnich, A.; Simonneau, G.; Humbert, M. Pre-implantation genetic diagnosis in pulmonary arterial hypertension due to $\mathrm{BMPR}_{2}$ mutation. Eur. Respir. J. 2012, 39, 1534-1535. [CrossRef]

9. Eichstaedt, C.A.; Song, J.; Benjamin, N.; Harutyunova, S.; Fischer, C.; Grünig, E.; Hinderhofer, K. EIF2AK4 mutation as "second hit" in hereditary pulmonary arterial hypertension. Respir. Res. 2016, 17, 1-8. [CrossRef]

10. Wang, G.; Knight, L.; Ji, R.; Lawrence, P.; Kanaan, U.; Li, L.; Das, A.; Cui, B.; Zou, W.; Penny, D.J.; et al. Early onset severe pulmonary arterial hypertension with 'two-hit' digenic mutations in both BMPR2 and KCNA5 genes. Int. J. Cardiol. 2014, 177, e167-e169. [CrossRef]

11. Viales, R.R.; Eichstaedt, C.A.; Ehlken, N.; Fischer, C.; Lichtblau, M.; Grünig, E.; Hinderhofer, K. Mutation in BMPR2 Promoter: A 'Second Hit' for Manifestation of Pulmonary Arterial Hypertension? PLoS ONE 2015, 10, e0133042. [CrossRef] [PubMed]

12. Hinderhofer, K.; Fischer, C.; Pfarr, N.; Szamalek-Hoegel, J.; Lichtblau, M.; Nagel, C.; Egenlauf, B.; Ehlken, N.; Grünig, E. Identification of a New Intronic BMPR2-Mutation and Early Diagnosis of Heritable Pulmonary Arterial Hypertension in a Large Family with Mean Clinical Follow-up of 12 Years. PLoS ONE 2014, 9, e91374. [CrossRef] [PubMed] 
13. Aldred, M.; Machado, R.D.; James, V.; Morrell, N.W.; Trembath, R. Characterization of theBMPR25'-Untranslated Region and a Novel Mutation in Pulmonary Hypertension. Am. J. Respir. Crit. Care Med. 2007, 176, 819-824. [CrossRef] [PubMed]

14. Wang, H.; Li, W.; Zhang, W.; Sun, K.; Song, X.; Gao, S.; Zhang, C.; Hui, R.; Hu, H. Novel promoter and exon mutations of the BMPR2 gene in Chinese patients with pulmonary arterial hypertension. Eur. J. Hum. Genet. 2009, 17, 1063-1069. [CrossRef]

15. Pousada, G.; Lupo, V.; Cástro-Sánchez, S.; Álvarez-Satta, M.; Sánchez-Monteagudo, A.; Baloira, A.; Espinós, C.; Valverde, D. Molecular and functional characterization of the BMPR2 gene in Pulmonary Arterial Hypertension. Sci. Rep. 2017, 7, 1923. [CrossRef]

16. Limsuwan, A.; Choubtum, L.; Wattanasirichaigoon, D. 5'UTR Repeat Polymorphisms of the BMPR2 gene in Children with Pulmonary Hypertension associated with Congenital Heart Disease. Hear. Lung Circ. 2013, 22, 204-210. [CrossRef]

17. Galiè, N.; Hoeper, M.M.; Humbert, M.; Torbicki, A.; Vachiery, J.-L.; Barbera, J.A.; Beghetti, M.; Corris, P.; Gaine, S.; Gibbs, J.S.; et al. Guidelines for the diagnosis and treatment of pulmonary hypertension: The Task Force for the Diagnosis and Treatment of Pulmonary Hypertension of the European Society of Cardiology (ESC) and the European Respiratory Society (ERS), endorsed by the International Society of Heart and Lung Transplantation (ISHLT). Eur. Hear. J. 2009, 30, 2493-2537. [CrossRef]

18. Nagel, C.; Henn, P.; Ehlken, N.; D’Andrea, A.; Blank, P.D.N.; Bossone, E.; Böttger, A.; Fiehn, C.; Fischer, C.; Lorenz, H.-M.; et al. Stress Doppler echocardiography for early detection of systemic sclerosis-associated pulmonary arterial hypertension. Arthritis Res. 2015, 17, 165. [CrossRef]

19. Grünig, E.; Janssen, B.; Mereles, D.; Barth, U.; Borst, M.M.; Vogt, I.R.; Fischer, C.; Olschewski, H.; Kuecherer, H.F.; Kübler, W. Abnormal Pulmonary Artery Pressure Response in Asymptomatic Carriers of Primary Pulmonary Hypertension Gene. Circulation 2000, 102, 1145-1150. [CrossRef]

20. Song, J.; Eichstaedt, C.A.; Viales, R.R.; Benjamin, N.; Harutyunova, S.; Fischer, C.; Grünig, E.; Hinderhofer, K. Identification of genetic defects in pulmonary arterial hypertension by a new gene panel diagnostic tool. Clin. Sci. 2016, 130, 2043-2052. [CrossRef]

21. Wang, J.; Zhang, C.; Liu, C.; Wang, W.; Zhang, N.; Hadadi, C.; Huang, J.; Zhong, N.; Lu, W. Functional mutations in $5^{\prime}$ UTR of the BMPR2 gene identified in Chinese families with pulmonary arterial hypertension. Pulm. Circ. 2016, 6, 103-108. [CrossRef] [PubMed]

22. Kim, Y.-S.; Nakanishi, G.; Lewandoski, M.; Jetten, A.M. GLIS3, a novel member of the GLIS subfamily of Kruppel-like zinc finger proteins with repressor and activation functions. Nucleic Acids Res. 2003, 31, 5513-5525. [CrossRef] [PubMed]

23. Eichstaedt, C.A.; Song, J.; Viales, R.R.; Pan, Z.; Benjamin, N.; Fischer, C.; Hoeper, M.; Ulrich, S.; Hinderhofer, K.; Grünig, E. First identification of Krüppel-like factor 2 mutation in heritable pulmonary arterial hypertension. Clin. Sci. 2017, 131, 689-698. [CrossRef] [PubMed]

24. Sindi, H.A.; Russomanno, G.; Satta, S.; Abdul-Salam, V.B.; Jo, K.B.; Qazi-Chaudhry, B.; Ainscough, A.J.; Szulcek, R.; Bogaard, H.J.; Morgan, C.C.; et al. Author Correction: Therapeutic potential of KLF2-induced exosomal microRNAs in pulmonary hypertension. Nat. Commun. 2020, 11, 1. [CrossRef]

25. Voz, M.L.; Agten, N.S.; Van De Ven, W.J.; Kas, K. PLAG1, the main translocation target in pleomorphic adenoma of the salivary glands, is a positive regulator of IGF-II. Cancer Res. 2000, 60, 106-113.

26. Nishi, H.; Nishi, K.H.; Johnson, A.C. Early Growth Response-1 gene mediates up-regulation of epidermal growth factor receptor expression during hypoxia. Cancer Res. 2002, 62, 827-834.

27. Gaddipati, R.; West, J.; Loyd, J.E.; Blackwell, T.; Lane, K.A. EGR1 is essential for transcriptional regulation of BMPR2. Am. J. Mol. Biol. 2011, 1, 131-139. [CrossRef]

28. Lopes, M.; Goupille, O.; Cloment, C.S.; Lallemand, Y.; Cumano, A.; Robert, B. Msx genes define a population of mural cell precursors required for head blood vessel maturation. Development 2011, 138, 3055-3066. [CrossRef]

29. GTExPortal Database Release V8. Available online: www.gtexportal.org (accessed on 8 March 2020).

(C) 2020 by the authors. Licensee MDPI, Basel, Switzerland. This article is an open access article distributed under the terms and conditions of the Creative Commons Attribution (CC BY) license (http://creativecommons.org/licenses/by/4.0/). 